This document is the accepted manuscript version of the following article:

Schneider, R., Losio, P.A., Nüesch, F.A. et al. Int J Adv Manuf Technol (2019).

https://doi.org/10.1007/s00170-019-03835-7

\title{
Gravure Printed Ag/conductive Polymer Electrodes and Simulation of their Electrical Properties
}

René Schneider ${ }^{1}$

Rene.Schneider@empa.ch; ${ }^{1}$ Laboratory for Functional Polymers, Empa, Swiss Federal Laboratories for Materials Science and Technology, Ueberlandstrasse 129, 8600 Dübendorf, Switzerland

Paolo A Losio $^{1}$

Paolo@losio.ch; ${ }^{1}$ Laboratory for Functional Polymers, Empa, Swiss Federal Laboratories for Materials Science and Technology, Ueberlandstrasse 129, 8600 Dübendorf, Switzerland

Frank A Nüesch ${ }^{1,2}$

Frank.Nueesch@empa.ch; ${ }^{1}$ Laboratory for Functional Polymers, Empa, Swiss Federal Laboratories for Materials Science and Technology, Ueberlandstrasse 129, 8600 Dübendorf, Switzerland

${ }^{2}$ Institut des matériaux, École Polytechnique Fédérale de Lausanne EPFL, Lausanne, CH-1015, Switzerland

Jakob Heier ${ }^{1}$

Jakob.Heier@empa.ch; ${ }^{1}$ Laboratory for Functional Polymers, Empa, Swiss Federal Laboratories for Materials Science and Technology, Ueberlandstrasse 129, 8600 Dübendorf, Switzerland

\section{E-Mail}

Rene.Schneider@empa.ch and Jakob.Heier@empa.ch

\section{Keywords}

Gravure printing, silver grid, metal mesh, transparent electrode, FEM Simulation

\section{Abstract}

In this paper some practical issues related to the manufacturing, and design criteria related to the application in devices of a hybrid silver grid / Poly(3,4-ethylenedioxythiophene) semi-transparent elec- 
trode are discussed. The electrodes are fabricated by gravure printing and screen printing. Experiments showed that defects in the printed grid due to imperfect ink transfer from the gravure roll to the substrate are detrimental to electrode performance. Various parameters like gravure cell design, printing speed or particle size of the ink were investigated to minimize the fraction of defects and to obtain highly conductive grids. It will be demonstrated, that overprinting of the lines is a feasible strategy to minimize the number of defects without noticeably broadening the lines.

While a defect-free grid is pre-requisite for such a hybrid device, for applications even stronger design criteria hold. That is addressed in the second part of the paper, where the electrical properties of the printed grids are simulated. With two exemplary device architectures, namely an organic light-emitting diode (OLED) and an organic photovoltaic cell, artificial load layers are integrated into the device structure and potential maps are calculated. The examples show how simulations can be deployed to design and optimize grid electrodes for a specific application. 


\section{Introduction}

In many thin film devices such as organic photovoltaic cells (OPV), organic light emitting diodes (OLEDs), light emitting electrochemical cells (LECs), displays and touch screens, transparent electrodes are an essential element. Indium tin oxide (ITO) coated glass is widely used as electrode, but its replacement is promoted since many years. Due to its lack of flexibility and bendability, ITO electrodes have limited applicability in flexible devices. Further drawbacks are the high price, scarcity of indium and time consuming multi-step processes to produce structured ITO electrodes. To replace ITO, a manifold of approaches, including metallic grids/mesh [1], conductive polymers [2], carbon nanotubes (CNTs) [3], silver nanowires [4], graphene [5], crack-nano-networks [6] or very thin metal layers [7] were developed [8]. All solutions strive for maximal transparency and high enough conductivity for the target application [9]. In spite of these research efforts, many approaches are only feasible on a small $R \& D$ laboratory scale and cannot be transferred to large scale production. Here solution processable materials which can be coated or printed have a major advantage, because these techniques are considered to be fast and cheap with high throughput for mass production.

Metal grids, gained much interest in the past years especially for touch screen applications, and manifold techniques are reported to produce them. Very thin silver (Ag) lines yielding virtually invisible grids can be manufactured by thermal imprint and filling [10], nano-imprint [11] and transfer printing [12].

Also a range of slower processes like aerosol-jet $[13,14]$, inkjet $[15,16]$ or lab scale printers utilizing offset [17], reverse offset [18], flexo [19] or gravure offset printing [20-23], can be used to print metal mesh structures with thin lines. Direct metal droplet printing, where laser [24], mechanical vibration [25] or air vibration [26] is used to generate metal droplets, is another potential technique to print metal structures. Non-printing techniques include for example self-assembly of nano-particles [27] or photolithography [28]. The importance of printing defect free lines is crucial for any of the techniques. Defects may result from manifold causes which are well-known from media printing [29]. Furthermore, defects also arise in non-conventional printing methods by air entrapment or incomplete flow filling [30-32].

Depending on the target application, the area between the printed metal mesh is filled with a transparent conductor like poly(3,4-ethylenedioxythiophene)-poly(styrenesulfonate) (PEDOT:PSS) [10] or a transparent conductive oxide (TCO) [33] to increase the uniformity of lateral conductivity.

By choosing the right printing technique, printed metal grids can provide both, a high conductivity at sufficient transparency and the potential for production on a (semi-) industrial scale. Direct gravure printing is a good candidate thanks to its precision retained even at fast printing speeds. Reports on direct gravure printing of thin conductive lines on an (semi-) industrial scale are scarce but they exist 
[34-36]. For a novel roller manufacturing method, namely diamond micro engraving, linewidths of 19 $\mu \mathrm{m}$ are reported [37]. The biggest problem encountered when printing thin conductive lines is the frequent appearance of defects, such as holes and interrupted pathways, affecting the conductivity of the printed line. Even on the laboratory scale, the printability of very thin lines can be a challenge [38, 39]. While gravure printing has a high potential for thin line printing, the necessary efforts on gravure cylinder design and ink formulation make it rather an academic exercise.

In this paper, conductivity measurements of gravure printed electrode silver grids including a conducting PEDOT:PSS filler layer, are combined with simulations of the electrical properties of the printed grids. Two aspects are considered here: 1 ) the overall resistivity of the grid as measured across the macroscopic sample, 2) the influence of the microscopic structure of individual grid cells (size, conductivity of filler film) on grid properties. The importance of printing defect free lines for a high overall film conductivity is demonstrated. Overprinting is introduced as an effective means to reach that goal. Furthermore, the strong variations in local conductivity along the period of the grid make an adaption of the grid properties to a specific application necessary. It will be demonstrated that an optimal grid cannot be defined by itself, but always depends on current and voltage characteristics of the application. Due to the specific needs of a particular device, the filler conductivity, linewidth, spacing, thickness and material of the metal mesh needs to be adapted.

Simulations can help to find an optimized architecture for a given application, including the conductivity of the filling layer.

\section{Material and Methods}

\subsection{Materials}

Two different silver inks, purchased from Genes' Ink were tested with gravure printing. The inks contain $5 \mathrm{~nm} \mathrm{Ag}$ (CS31506) and $20 \mathrm{~nm}$ Ag particles (S-CS51301), respectively, these inks will be denominated "5 nm ink" and "20 nm ink", respectively. The PEDOT:PSS layer was printed with a screen printing ink purchased from AGFA (Orgacon EL-P 3145). Bus bars were painted with Ag conductive paint (RS Pro) using a swab. Different substrates, including PET (CF-1 new quality, covered with a thin ink receptive coating for better ink-adhesion; $130 \mu \mathrm{m}$ thickness) by Folex AG, Switzerland, thin glass AF32 eco (50 $\mu \mathrm{m}$ thickness) by Schott AG, Germany, and a cellulose triacetate (CTA) foil ( $80 \mu \mathrm{m}$ thickness) provided by Folex AG were used. Additionally, a thin glass-PET laminate (100 $\mu \mathrm{m}$ thickness) was used, here only the glass side was used for printing. All substrates were cleaned with Isopropanol and dried with dustfree compressed air before use. Substrates were placed on a printing blanket (Rollin; type 100 by Trelleborg; $1.00 \mathrm{~mm}$ thickness) for printing.

\subsection{Printer}


Printing was performed on the multifunctional sheet-to-product printer $\mathrm{C} 600$ manufactured by nsm Norbert Schläfli AG, Switzerland, hosting a gravure-, flexo- and screen printing unit, as well as a hot air Comb Nozzle ${ }^{\circledR}$ dryer from $C N$ drying technology, Germany. The high repeat accuracy of the nsm C600 was demonstrated by measuring marker positions of repeatedly printed markers. As shown in Figure $S 1$, the markers' position doesn't change within a range of approximately $\pm 5 \mu \mathrm{m}$ after overprint. Especially the gravure unit showed a very high repeat accuracy, namely $\pm 3 \mu \mathrm{m}$ in $\mathrm{x}$ - (= printing direction) and $\pm 2 \mu \mathrm{m}$ in $\mathrm{y}$ - direction. Thus, the printing lateral resolution is sufficient for printing multiple layers and thin lines on top of each other.

\subsection{Gravure printing and cylinder design}

For gravure printing, a laser engraved (engraving by GRT GmbH, Germany) printing roller with three different cell depths (see Table 1) was used. Solid lines were drawn at the resolution of the cells. Lines of the grid cross to form a diamond shape.

For printing, the doctor blade pressure was set to 6.9 bar, further printing parameters are listed in Table S1. For multilayer prints the Ag ink was dried between each printing step at $80^{\circ} \mathrm{C}$ for 90 seconds. After all layers were printed, the samples were sintered in an oven at $150^{\circ} \mathrm{C}$ for 30 minutes to achieve maximum conductivity.

\subsection{Screen printing}

For screen printing, a standard PET mesh $\left(165 / 30 ; 22.5^{\circ}\right)$ was used. Lift-off was set to $0.800 \mathrm{~mm}$, the printing speed was $100 \mathrm{~mm} / \mathrm{s}$ and the squeegee angle was $71.5^{\circ} \pm 0.5^{\circ}$.

\subsection{Ag mesh - PEDOT:PSS-hybrid}

The Ag mesh was printed on respective substrates as specified above. Ag printing was repeated up to three times. After sintering all samples were covered with 2 layers of screen printed PEDOT:PSS to achieve complete coverage of the Ag grid. Samples were placed on a hotplate set to $100^{\circ} \mathrm{C}$ for 10 minutes to improve conductivity by annealing [40] and to remove water residuals.

\subsection{Microscopy / Surface Metrology}

To obtain information on the surface morphology and line thickness, a non-destructive, confocal 3D Optical Surface Metrology System (DCM8, Leica Microsystems AG, Switzerland) was used. Images were taken with Leica EPI 5x or EPI 20x objectives, while thickness measurements were performed with a Mirau 20x objective in a phase-shift experiment (PSI) for high z-resolution. Data were analyzed with the Leica Map 7.2 software to gain line cross-section and line thickness. At least 25 cross sections were measured to obtain an average value.

\subsection{Resistivity measurement}

We define the "resistivity of the sample" as the absolute resistivity that is obtained using the measurement geometry depicted in Figure 7(a). Resistivity measurements for samples used for graphs in this 
publication were measured with a home built 4 point probe using a Keithley 2400 source meter (Cleveland, United States). Other samples were roughly checked with a multimeter. This resistivity can only approximatively be related to a sheet resistance, as discussed in the results section.

\subsection{UV-VIS}

Transmission spectra were measured on a Varian Cary 50 UV-VIS spectrometer (Agilent Technologies, (Santa Clara, United States)).

\subsection{Simulations}

Simulations using the finite element method (FEM) were performed using the freely available multiphysics FEM package NM-Seses developed at ZHAW [41] and a Matlab control script denominated GridCalc adapted from previous work. $[42,43]$ The software tool can be requested from the corresponding author. Using the FEM Gridcalc allows to accurately calculate the potential distribution and the current flow $\mathrm{J}$ in an extended planar device comprising metallic grids and conducting filler materials. The planar materials are treated as 2D domains and several planar materials can be interconnected by perfect contacts. Modeling the planar conductors as $2 \mathrm{D}$ domains implicitly makes the assumption of a constant thickness of the conductor across the whole surface, furthermore the conductivity is assumed to be constant along the layer thickness. Although these assumptions may be too simplistic, they allow for efficient computing. Additionally, it is possible to add a further planar conductor and connect it to the previous structure through a load component. A schematic drawing of the geometries is shown in Figure 1.

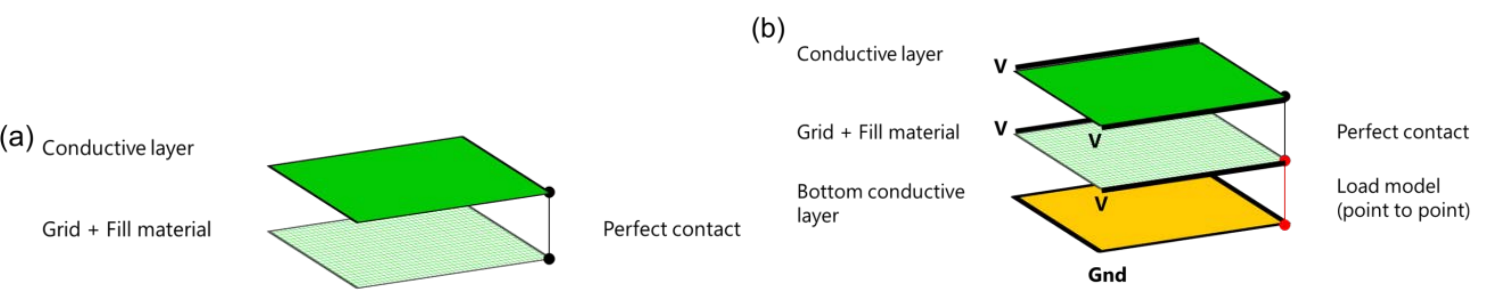

Figure 1: Schematic drawing of the simulation geometry for model without (a) and with load layer (b).

The load component can be modeled using a simple linear law $(J=(1 / R) \times V)$, a power law $\left(J=A \times V^{B}+C\right)$ or an exponential law $(J=A \times \exp (B \times V)+C)$ defining the current flow $J$ as a function of the potential difference $V(R=$ resistivity; $A, B, C=$ fitting parameters). Modeling a load allows to understand if a grid design is suitable to drive extended planar devices like organic light emitting diodes or to efficiently collect current from photovoltaic thin film devices. To model the potential distribution and current flow across electrodes, Dirichlet boundary conditions are applied at the relevant boundaries corresponding to the experimental conditions and all other boundaries are defined as insulating. Conductors are defined by the experimentally determined conductivity and measured average layer thickness. The geometry of metallic meshes can be synthetically generated in the software or can be imported from 
bitmapped microscope images to correctly model printed meshes including their defects and variations.

Even using a planar approximation, it is computationally expensive to model a full metallic grid with line widths of a few $\mu \mathrm{m}$ covering several $\mathrm{cm}^{2}$ of surface due to the large number of resulting points. The problem can be solved by adopting a stepwise approach. In a first step, denominated "micro" simulation, the conductivity of a printed grid is obtained by simulating a surface of few square millimeters consisting of a printed grid at high resolution and filler material. In the second step, denominated "macro" simulation, the conductivity of the mesh and filler system obtained in the first step is used as filler material in the simulation of a larger electrode. In this case the metallic grid represents a large scale electrode, busbar or similar structure and the filler material is the composite printed mesh. Exemplary structures for "micro" and "macro" are shown in Figure 6 and Figure 7 (a), respectively.

The used input parameters were: $\mathrm{Ag}(5 \mathrm{~nm}$ ink; CS31506)): 2.9×10^7 S/m*; Ag (20 nm ink; S-CS51301): 8.4×10^6 S/m*; PEDOT:PSS: $135 \mathrm{~S} / \mathrm{m}^{*}$; Ag paint $789889 \mathrm{~S} / \mathrm{m}^{* *}$ ( ${ }^{*}$ manufacturer value; **measured). Thicknesses were measured either by microscopy or calculated from cross-sections of printed lines (obtained from microscopy) divided by the line width obtained from the input image. For ohmic load calculations the exponential law was used with $A=0.9, B=1.12 \mathrm{~V}^{-1}, C=0.9 \mathrm{Am}^{-2}$ for OLED characteristics and $A=0.12 ; B=9 V^{-1} ; C=-200 \mathrm{Am}^{-2}$ for $P V$ type characteristics. $A, B$ and $C$ were chosen to fit experimental data found in literature $[44,45]$.

\section{Results and Discussion: Printing}

Gravure printing requires the optimization of a number of correlated printing parameters. Five different grids (A-E) with different pitch and different gravure cell depths were tested. Values are listed in Table 1. On the gravure cylinder design side, engraving depth and nominal line width influence the amount of ink that can be transferred to the substrate. That in turn determines line width and conductivity of individual lines, and is also critical in the formation of defects. Pitch (grating periodicity) largely determines the overall sheet resistance of the substrate, we will see later to what extend a large pitch can be compensated by the PEDOT:PSS filler. In addition, the gravure roll-to-substrate distance (gap) was varied. Gravure roll-to-substrate - distance is an indirect measure for roll pressure which cannot be monitored directly in our setup. Values are given in Table S1 (in the supplementary information section) calculated by $\Delta$ gap = gap - substrate, with higher values meaning higher pressures. 2 different inks, which contain either $5 \mathrm{~nm}$ or $20 \mathrm{~nm}$ particles were tested on various substrates like PET, ultrathin glass and CTA. As last parameter, also the printing speed is varied between 300 and $1200 \mathrm{~mm} / \mathrm{sec}$, indicating the industrial relevance of gravure printing.

Table 1 Gravure cylinder parameter for 5 different grid geometries (A-E)

$\begin{array}{lllllll}\text { Grid } & \text { A } & \text { B } & \text { C } & \text { D } & \text { E }\end{array}$




\begin{tabular}{llllll}
\hline Pitch $[\boldsymbol{\mu m}]$ & 290 & 580 & 290 & 580 & 580 \\
\hline Engraved (nominal) line width $[\boldsymbol{\mu m}]$ & $10(10)$ & $10(10)$ & $12^{*}(10)$ & $12^{*}(10)$ & $15^{*}(10)$ \\
\hline Gravure cell depth $[\boldsymbol{\mu m}]$ & 8 & 8 & 16 & 16 & 24 \\
\hline Theoretical cell volume $\left[\mathbf{c m}^{3} / \mathbf{m}^{2}\right]$ & 6.0 & 6.0 & 11.7 & 11.7 & 15.7 \\
\hline
\end{tabular}

*Estimated line width. The actual engraved line width is larger than nominal due to diameter of the laser beam used for cylinder engraving.

The print quality of the grids was evaluated using the following three criteria:

1. Fraction of defects: Number of defects identified by microscopy divided by the number of intersection points of the grid in the scanned area. Since one line can have more than one defect, the fraction of defects can theoretically be $>1$. Typical defects, consisting of interrupted lines are shown in Figure 2(b).

2. Resistivity of the grid. This is the total resistivity of the grid as measured across the macroscopic sample.

3. Line width and optical transmittance.

In Figure 2(a) the fraction of defects and resistivity data for the five grid geometries printed with $20 \mathrm{~nm}$ ink is shown. Even though there is no clear trend in number of defects versus grid conductivity due to scatter in data and the large number of parameters probed, one conclusion can be drawn: to obtain low resistivity, the number of defects must be low. Similarly, a high number of defects typically results in high resistivity. Next to that, the data reveal some more detailed information: Figure 2(a) shows that the print quality decreases with increasing printing speed for $20 \mathrm{~nm}$ ink. The fraction of defects is increasing, while the resistivity also increases at higher speed. Thus a low printing speed is beneficial when using $20 \mathrm{~nm}$ particle ink.
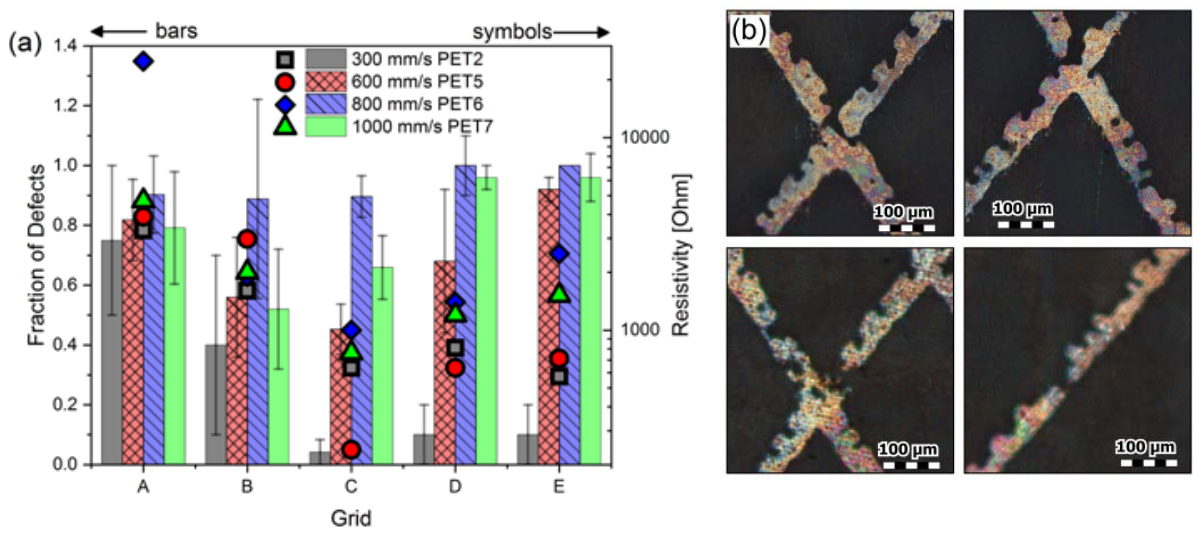

Figure 2(a): Fraction of defects (bars) and corresponding resistivity (related to particular sample geometry, data points) for 5 different grid geometries (A-E) of one layer (PET2, PET5, PET6, 
PET7) samples. Different printing speeds were tested using the $20 \mathrm{~nm}$ ink. According to the definition of number of defects, a fraction of 1 corresponds to every second line interrupted. Error bars indicate the fraction of potential defects which could not be definitely identified as interrupted pathways like submicron gaps etc.. (b): Typical defects showing interrupted pathways obtained from sample PET14. Narrowing of lines is not counted as a defect.

Figure 2 (a) also shows that gravure cell design has an influence on the grid quality, but only in conjunction with the printing speed. That is in agreement with earlier reports that the process of cell emptying depends on cell design and printing speed [46]. In our case grid C usually shows best results, and this trend is true for nearly all one layer prints on PET substrate, which leads to the conclusion that a gravure cell depth of $16 \mu \mathrm{m}$ is somehow an optimum for the examined system. Thus a midrange depth and small pitch give the best results. Also that makes perfectly sense considering that conductivity relates to the amount of silver ink transferred to the substrate (cross section of printed lines is shown in Figure S3).

The same trend is observed for smaller Ag nanoparticles, even though less distinctly. Also a gravure roll-to-substrate-distance variation did not affect the resistivity data much. Samples showed more or less the same values for grids of each grid-type (A, B and C; see Figure 3).

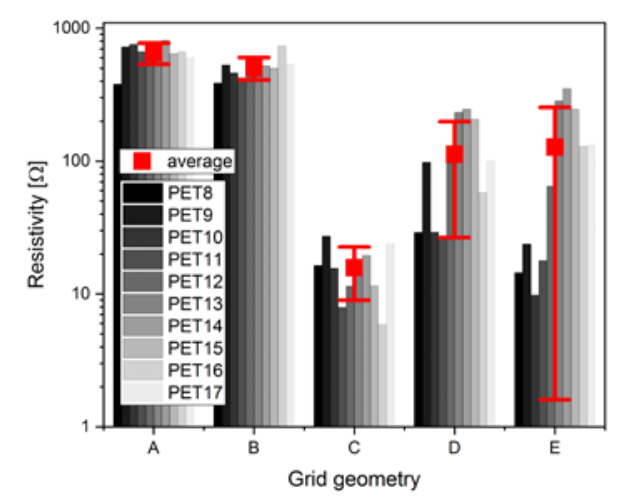

Figure 3: Average resistivity for $5 \mathrm{~nm}$ ink samples at various printing speeds (see Table S1 for details).

Compared to the $20 \mathrm{~nm}$ ink, resistivity is much smaller when the $5 \mathrm{~nm}$ ink is used, but still not satisfying our defined target.

To sum up, $5 \mathrm{~nm}$ ink is easier to print and gives better, but not yet satisfying results on conductivity. For simple one layer printed grids, a resistivity below an arbitrary chosen threshold value of $<50 \mathrm{Ohm}$ could not be obtained. This can be traced back to interrupted pathways (main defects), which decrease possible current paths and thus increase overall resistivity of the grid.

The appearance of defects during line printing was here identified as one hurdle for the realization of a gravure printed electrode grid. 
In inkjet $[47,48]$ and screen printing $[49]$, overprinting of the lines was suggested as solution, to the best of our knowledge it has not yet been reported for gravure printing. In Figure 4, the fraction of defects and resistivity of twice and three times overprinted grids are compared to the single prints. The number of defects and the resistivity of the grids decrease remarkably due to overprint.
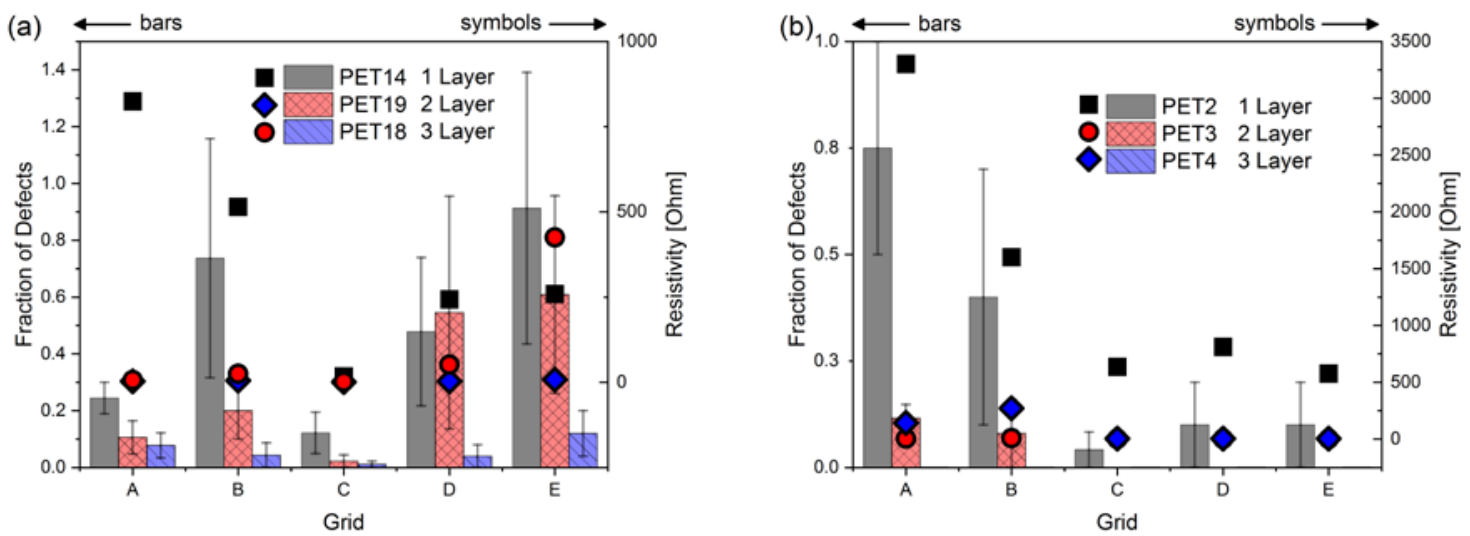

Figure 4(a): Fraction of defects (bars) and corresponding resistivity (related to particular sample geometry, data points) for 5 different grid geometries (A-E) of one, two and three layer (PET14, PET19, PET18) samples, printed with $5 \mathrm{~nm}$ ink. (b): Fraction of defects (bars) and corresponding resistivity (related to particular sample geometry, data points) for 5 different grid geometries (A-E) of one, two and three layer (PET2, PET3, PET4) samples, printed with $20 \mathrm{~nm}$ ink.

The additionally deposited Ag will fill holes and reconnect interrupted pathways. It could also be shown that the width of the lines did not increase (Table S2). The success of the method is facilitated by the overprint accuracy of the printer C600 (Table S2).

Optical transmittance is introduced here, as it is a key measure for an acceptable linewidth. Data for three layer samples of $5 \mathrm{~nm}$ ink and $20 \mathrm{~nm}$ ink on PET substrates, as well as transmittance data of plain substrates are shown in Figure 5(a) and (b), respectively. As expected, grids with larger linewidth and smaller pitch show lower resistivity but also lower transparency. Despite the larger linewidth for $20 \mathrm{~nm}$ ink, only a slight difference between $5 \mathrm{~nm}$ ink (symbol) and $20 \mathrm{~nm}$ ink (dashed line) in transparency is visible for grids $A$ and $B$. Using the $5 \mathrm{~nm}$ ink, an intense toning of the non-printed areas of the PET substrate was observed. This is a common problem with inks consisting of very small nanoparticles. Due to their small size, the lubrication film which is always present during the gravure printing process contains also particles from the ink. [46] This ink is transferred from the non-printing areas of the cylinder to the substrate. In addition, a worn or misaligned doctor blade and/or a certain roughness of the cylinder promote this problem. Changing the ink to $20 \mathrm{~nm}$ Ag particle size, toning of the nonprinted areas also decreased for the PET substrate. Optical transmittance of the non-printed area (adjacent to the mesh) increased by $10-20 \%$ as shown in Figure 5(a). In this case the particle size may be too large to cross the gap underneath the doctor blade. 
Toning of the PET substrate may be attributed to the thin ink receptive coating of the PET substrate, but this was not further investigated.
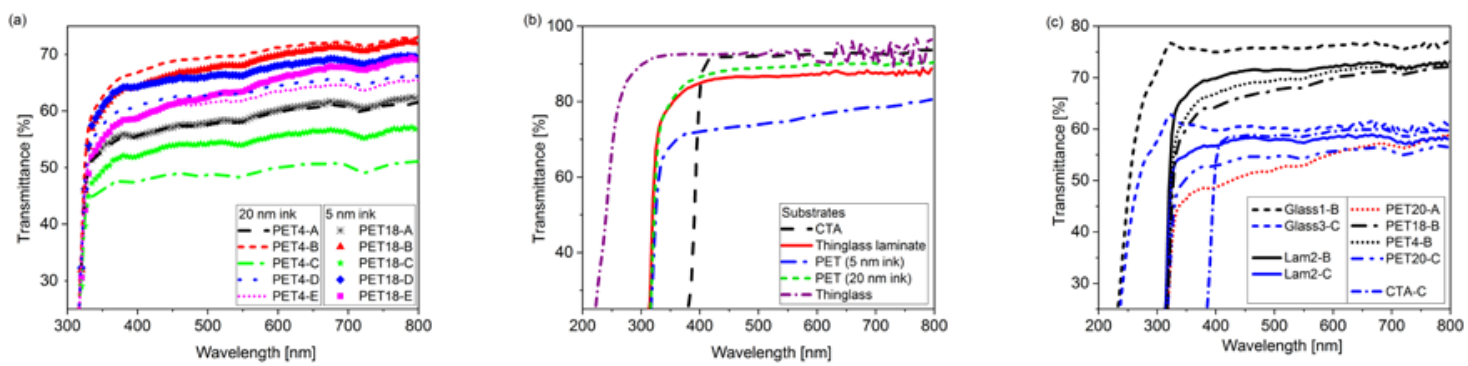

Figure 5(a): UV-VIS transmittance of 3 layer samples printed on PET substrate for $\mathbf{5} \mathbf{~ n m}$ and 20 nm ink and different grid geometries (A-E). (b): UV-VIS transmittance of the used substrates (c): UV-VIS transmittance of best performing samples.

\section{Best performing samples}

Low resistivity samples can still be optimized with respect to transmittance by using different substrates. Data for these samples are shown in Figure 5(c). Samples having flexible thin glass as substrate show highest transmittance of approximately $75 \%$ for printed grid B with a cut-off at approximately $250 \mathrm{~nm}$. The handling of these samples is rather delicate and samples break easily even by touching edges due to the brittleness of the thin glass. Thus a thin glass-PET laminate, which is much more robust, was used as substrate. A PET layer laminated at the backside of the glass increases robustness of the sample drastically, while all glass advantages such as the absence of toning and very low surface roughness are retained. As a result of the additional PET layer, the transmittance is decreased by approximately $5 \%$ and cut-off is shifted to $310-320 \mathrm{~nm}$. Compared to pure PET substrates, the transmittance is higher due to less toning. Also, the $5 \mathrm{~nm}$ ink did not show toning if ultrathin glass, glass laminate or CTA were used as substrate. In all cases optical transmittance is well above $80 \%$ and for flexible glass with $50 \mu \mathrm{m}$ thickness the transmittance was approx. $92 \%$. In summary the $5 \mathrm{~nm}$ particle ink printed on ultrathin glass yielded best performing samples.

\section{Results and Discussion: Simulation}

By simulating the electrical properties of the grid electrodes, information that is otherwise not easily accessible can be gained. In applications where the electrode is used as a top electrode, the voltage drop within a single cell between the silver lines is of uttermost importance and cannot be measured easily. On the other hand, the measured resistances can be simulated. We here compare measured and calculated grid resistivities to validate the model. Input for "micro" simulations is a b/w bitmap of an actual microscope image of a printed Ag grid. The threshold is chosen such that Ag and non-Ag areas are well differentiated with black and white, representing two different conductive materials in the first 
layer (Figure 6 left images). Black pixels are set to have Ag like conductivity $\left(2.9 \times 10^{7} \mathrm{~S} / \mathrm{m}\right)$, while white areas have PEDOT:PSS like conductivity $(135 \mathrm{~S} / \mathrm{m})$. Also a thin continuous planar layer with PEDOT:PSS conductivity is incorporated. The left edge of the input picture is set at OV (GND) while edge on the right is set to $+1 \mathrm{~V}$ operating voltage, which corresponds exactly to the measurement geometry. With this setup it is possible to model the printed grids considering printing defects and finally calculating their resistivity.

In the right images of Figure 6 the calculated values of the resistivity are indicated. The graphs give a qualitative representation of ohmic losses which are expressed as relative ohmic losses (or Joule Heating, equal to the product of the square current density and the resistivity). Results are scaled from 0 to 1 with a corresponding color code from black to white.

As shown in Figure 6, resistivity decreases with the number of layers of printed Ag. This is in accordance with experimental measurements: by performing multilayer prints the layer thickness increases and the fraction of defects decreases. By comparing each pair of pictures, it can be observed that defects in the printed grid lead to zero current flow, so that branches of the grid are drawn in black or dark red. Additionally, when grid branches are interrupted, the current flows through other branches resulting in lighter color due to a locally increased current flow. Results also show that PEDOT:PSS filled areas between the Ag grid conduct only very little current due to poor conductivity, and thus can also only show very small ohmic losses.

We can draw the important conclusion that filling of the gaps with PEDOT:PSS doesn't effectively bridge interrupted pathways. . The simulation results suggest that an increase by two orders of magnitude in conductivity would be needed to show a significant impact of PEDOT:PSS on the overall grid resistivity. Thus, when a voltage is applied in plane of the sample, the main fraction of current is flowing through the Ag grid. 


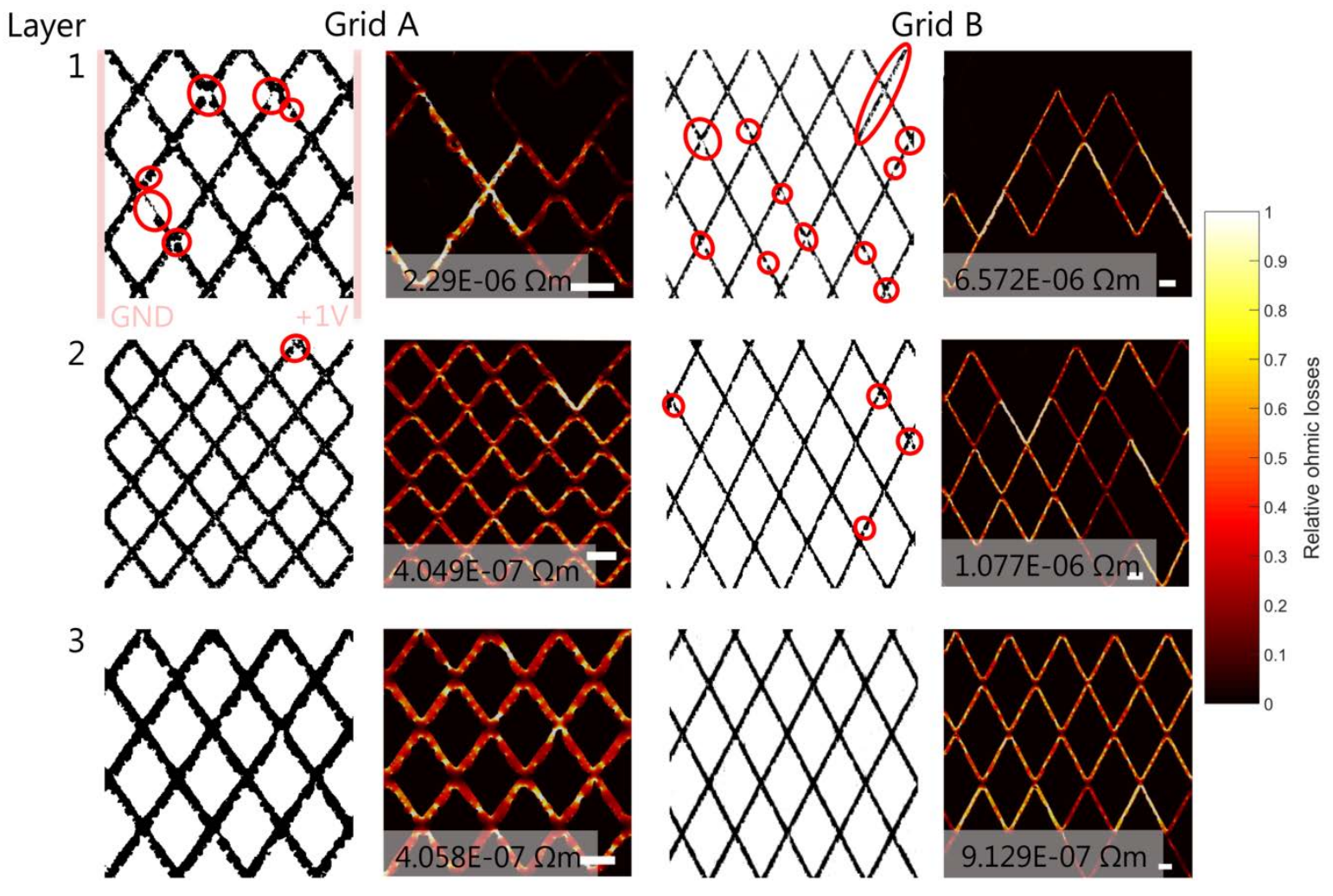

Figure 6: Input bitmap pictures and simulation results for grid geometry A and B with 1, 2 and 3

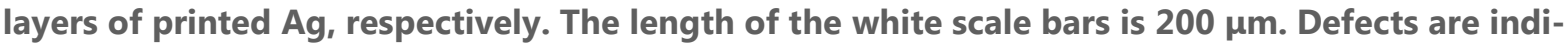
cated with red circles; Results: color code shows relative ohmic losses. Samples used: PET14, 18, 19.

\subsection{Comparison with experiment}

In the experimental set-up the voltage is applied at two contact points at bus bars contacting the grid (Figure 7(a)). One can see that a voltage drop along the bus bar has an influence on the measured grid resistivity. For that reason, the "macro" simulations were extended to reflect the exact measurement geometry and determine the average resistance $R_{\text {average }}$ from there (Figure $7(a)$ ). The white area is set as average conductivity from the "micro" simulations and grey areas represent the bus bars. Contact points at GND and $+1 \mathrm{~V}$ are indicated black and red, respectively. Simulation yields a voltage distribution and an average current and average resistivity for the given sample geometry. An exemplary result is shown in Figure 7(b) for sample PET18-C. 

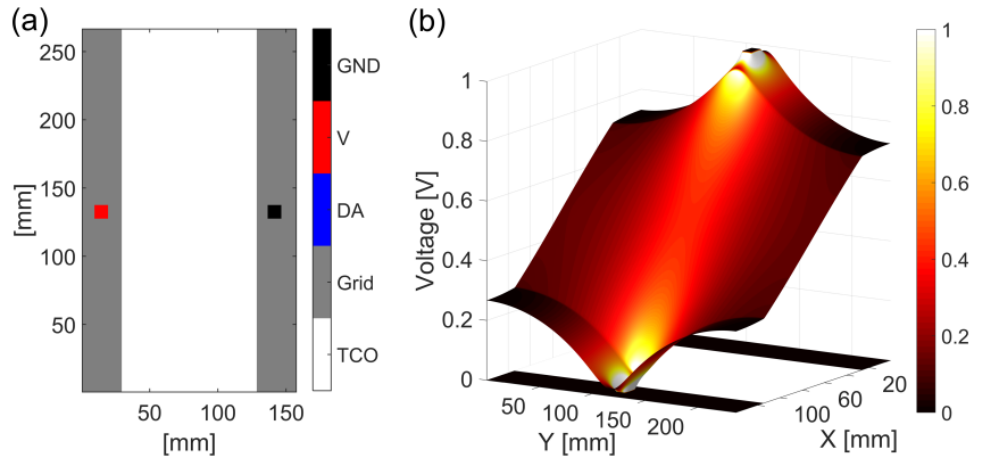

Figure 7(a): Sample geometry for large samples with GND = ground; V = 1V; DA = optional dead area (not used here); Grid = Ag bus bar; TCO = printed grid. (b): Simulation result for PET18-C showing voltage drop across the sample as well as voltage loss within the bus bars. The color code indicates relative ohmic loss.

In this case a voltage drop along the busbar is observed, which is due to the limited conductivity of the used Ag paint to draw the bus bar. Due to this non-ideality it is not possible to calculate a sheet resistance for the macroscopic sample.

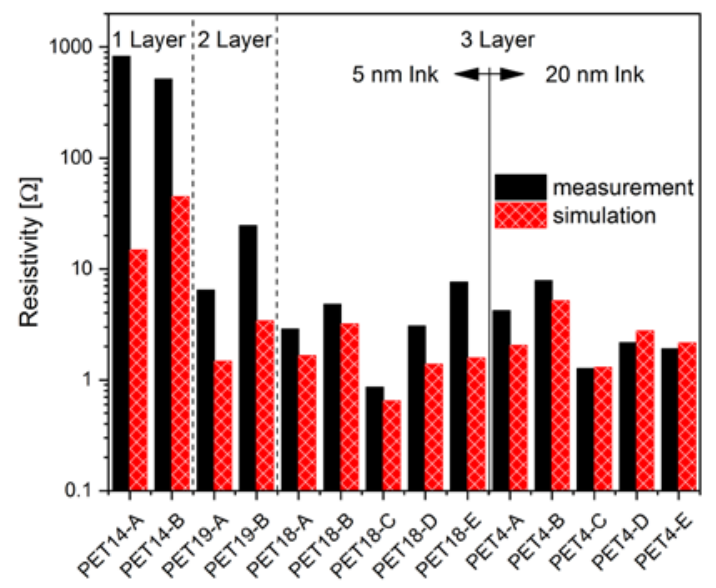

Figure 8: Comparison of resistivity values for experiment and simulation

Different grids with various parameters were simulated and average resistivity results are shown in Figure 8 and compared to the measured resistivity of the samples. Figure 8 also shows a huge discrepancy between simulated and measured resistivity for one layer prints, where the simulation drastically overestimates conductivity. For two layer prints discrepancy in resistivity is also high, but decreases compared to one layer prints, while for three layer prints simulation and experiment show passable agreement. The experimental trend that grid $C$ showed highest conductivity for any dataset and that a gravure cell depth of $16 \mu \mathrm{m}$ is somehow an optimum for the examined system is confirmed by simulations for grid C of sample PET18 and PET4. We can summarize that simulation and experiment only co- 
incide satisfyingly for low resistive samples. The discrepancy between simulated and measured values for high resistive samples may have various reasons:

Besides interrupted lines, which are taken into account as defects, there might be additional submicron sized defects and interruptions which cannot be detected by the microscope. Thus the real number of defects is actually higher. Assuming that the number of detected and the number of undetected defects show a similar behavior, this discrepancy could be used as a measure for hidden and undetected defects.

Secondly, the narrowing of lines due to improper ink transfer in one or two layer grids reduces the overall conductivity of the grid significantly. In the experiment a narrow section of a line typically also shows a reduced height, while the simulations assume that all lines and connections have the same height. Thus the simulations underestimate the resistivity of these locally thinner connections leading to a lower simulated sample resistivity.

Thirdly, one of the most critical input factors for the simulations is the average Ag grid thickness. As Ag conductivity is constant, the thickness affects the amount of material and thus the resistivity of the grid. High resistive samples (e.g. one, or two layer grids) have thinner layers and thus an overestimated layer thickness affects these grids more, leading to a underestimated resistivity in the simulation. This is expected to be a minor effect, because thickness measurement was carried out carefully. But gravure printed thin lines have a large thickness variation and roughness, making thickness measurement a non-easy task with a high standard deviation. For example, the roughness $R_{q}$ of a printed line is already almost half of the line's average thickness $D(R q=27 \mathrm{~nm} ; D=57 \mathrm{~nm}$; determined for Grid $A$; sample PET14).

In summary, simulation and experiment agree well for highly conductive samples, while low conductive samples show much higher resistivity than expected from the simulations.

\subsection{Case study}

Previous reports on the use of silver grids as transparent electrodes in devices were focused on the correlation between sheet resistance and transmittance. Of similar importance in device applications are losses due to the voltage drop between the Ag-lines. This effect was analyzed by simulating the voltage distribution in the printed grid layer when an artificial load layer is sandwiched between Aggrid and an ideal back-electrode. The model layout follows a typical thin film device: the bottom contact is a highly conductive homogeneous layer set at $0 \mathrm{~V}$. The top contact is the printed grid electrode whereby two edges are set to the operating voltage $+\mathrm{V}$. A load-layer is sandwiched between those layers. The load-layer is conductive in z-direction and a constant, a linear or an exponential (approximating a diode) I-V-dependence can be assigned to the load layer. Hence it is possible to investigate the behavior of printed grid for various device architectures. 
Two scenarios are presented, in the first case a consumer with a typical OLED-diode characteristics was introduced. In the second case the "load-layer" is formed by a photovoltaic material generating a photocurrent. In both cases, the simulation calculates the voltage over the printed grid and represents it in a 3-D dataset.

The grid geometry, conductivities of Ag and PEDOT:PSS, and J-V-characteristics of the load-layer determine the voltage changes between the Ag lines (diamond shaped areas in this samples are referred to as combs). The lowest value is located in the center of a comb, with maximal distance from the Aglines and maximal voltage loss $(\Delta \mathrm{V})$. This voltage distribution can be used to determine the suitability of a certain grid for a certain application.

In the OLED case, a typical superyellow-OLED J-V curve, was used as load for simulations and operated at $7 \mathrm{~V}$ [44]. The maximum voltage loss $\Delta \mathrm{V}_{\max }$ is shown in Figure 9 (a) as a function of grid pitch for three different PEDOT:PSS conductivities. As reference, a high conductive grade PEDOT:PSS (135 S/m; red circular data points; AGFA Orgacon EL-P 3145) was used. From this starting point, conductivity was increased or decreased by two orders of magnitude, respectively. These conductivities may not be reached in real systems and may not have practical application, but help to gain deeper insight in the effect of large or small conductivity contrast between grid and filler. As expected, voltage loss $\left(\Delta \mathrm{V}_{\max }\right)$ increases with increasing pitch, as well as with decreasing PEDOT:PSS conductivity.

Decisive for a grid design for an OLED application is the high sensitivity of the human eye to intensity changes, but also its constraint to resolve high resolution features. In the best case, intensity changes by $2 \%$ can be noticed [50]. Following the J-V curve, a voltage drop of only $10 \%$ (e.g. from $7 \mathrm{~V}$ to $6.3 \mathrm{~V}$ ) would lead to a decrease of luminescence to $66 \%$. On the other hand, e.g. the pixel pitch in a monitor with a standard 72 PPI resolution corresponds to a pixel size of $353 \mu \mathrm{m}$. Thus for our smallest pitch of $290 \mu \mathrm{m}$ the luminance inhomogeneity will merge for a human eye, only affecting the overall brightness.

Voltage distributions are shown in Figure 9(b) for samples with PEDOT:PSS conductivity of $135 \mathrm{~S} / \mathrm{m}$ and varying grid pitch (corresponding data points are marked with (1), (2), (3)). The cut-off at $\Delta \mathrm{V}=$ $0.7 \mathrm{~V}$ indicates that already a large part of the comb-area has a significantly reduced luminescence in the center area of a comb. As PEDOT:PSS conductivity decreases, the voltage loss slope becomes very steep and the dark spot area increases rapidly. For the larger pitches homogeneity to the human eye is thus very difficult, if not impossible to maintain. In an OLED application the grid pitch must correspond to the desired lateral resolution. 
(a)

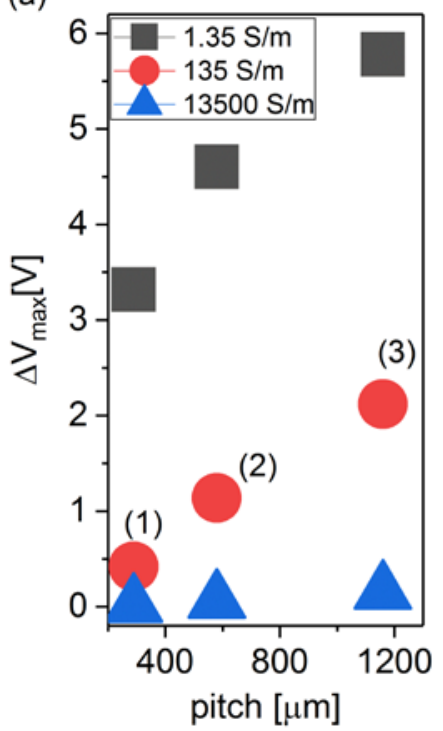

(b)

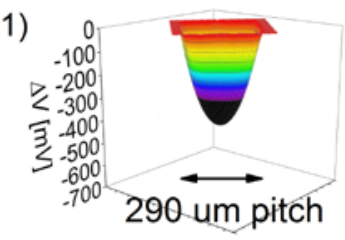

(2)

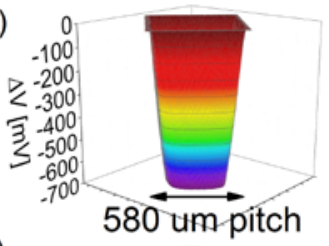

(3)

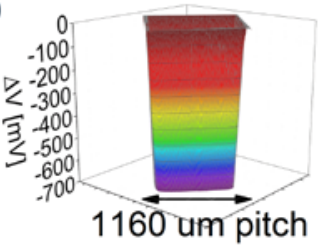

Figure 9(a): Maximum voltage loss at center of comb ( $\Delta \mathbf{V}_{\max }$ filled symbols) as a function of grid spacing for 3 different PEDOT:PSS conductivities. (b): Voltage distribution for PEDOT:PSS conductivity of $135 \mathrm{~S} / \mathrm{m}$ and small (1), medium (2) and large (3) pitch, respectively. Cut-off is performed at loss of $700 \mathrm{mV}$, when luminance dropped to $66 \%$ of its initial value.

For PV type characteristics, an approximated perovskite solar-cell J-V curve, operating at maximum power point (here $0.654 \mathrm{~V}$ ) was used as input for the simulations. [45] In this case the simulation results are similar to the OLED case showing a voltage variation over the grid. For PV type applications the more relevant information is the power output of the cell, dropping to zero when the voltage drops to 0. In Figure 10(b) the power distributions for small pitch at two different PEDOT:PSS conductivities are shown. For higher conductive PEDOT:PSS, the power drops by $7 \%$ at the center of a comb. If the PEDOT:PSS conductivity is lower, the power turns negative at the center of a comb, showing, that the PV cell is not working at all in that area. In fact the dead area ( $P \leq 0)$ of this system is around $15 \%$ of the comb area. In real systems also shadowing by the printed Ag mesh should be taken in account, which is not done in the simplistic approach presented here. Figure 10(a) shows the averaged power $P_{\text {average }}$ over the whole comb for the simulated samples, as well as the dead area (open symbols) and, as expected, power decreases with increasing pitch. 

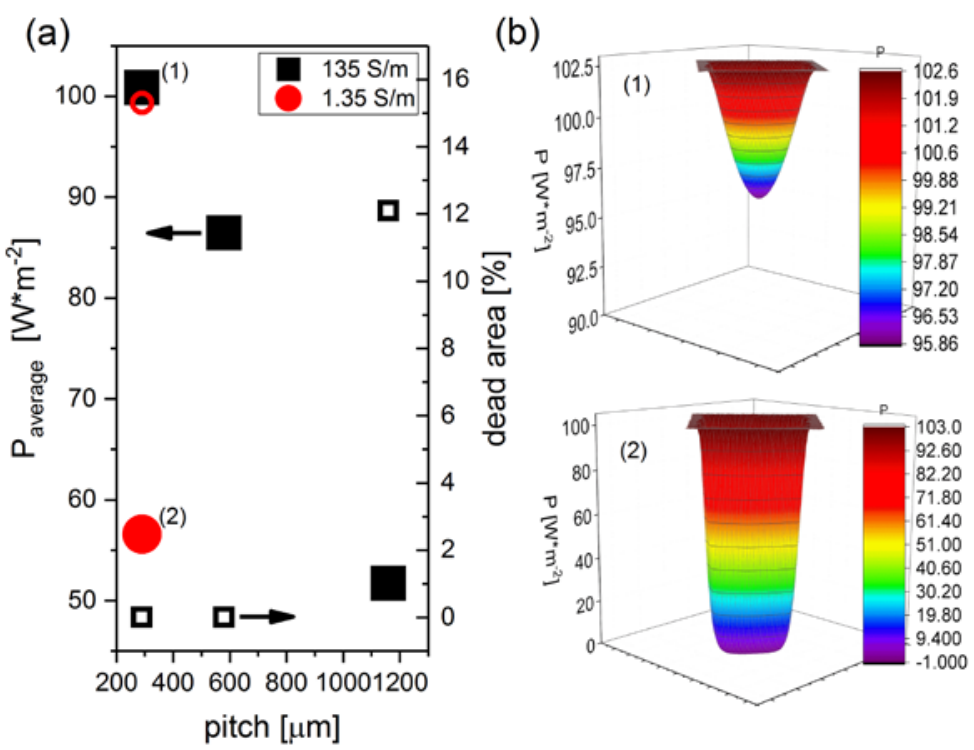

Figure 10(a) Simulation result with a PV type characteristics of the device load. Averaged power ( $P_{\text {average, }}$ filled symbols) over the whole comb, as well as the dead area (open symbols) as a function of grid pitch is shown. (b): Power distribution for combs with small pitch and high (1) or low (2) conductive PEDOT:PSS. P $<0$ indicates areas that cannot contribute to PV activity.

\section{Conclusion}

In the first part of the paper we discussed some practical issues related to the manufacturing of a hybrid-silver grid PEDOT:PSS semi-transparent electrode by gravure printing and screen printing.

Detrimental to electrode performance are defects in the printed grid due to imperfect ink transfer from the gravure roll to the substrate. Experiments showed that various printing parameters affect the number of defects of the printed grids and thus the grid conductivity. It was shown that gravure cell design has an influence on the grid quality, but only in conjunction with the printing speed. With two inks with different nanoparticle size conductive grids were successfully printed. The $5 \mathrm{~nm}$ ink was easier printable and gives better, but not yet satisfying results on conductivity. It was demonstrated that overprinting of the lines is a feasible strategy to decrease the fraction of defects without noticeably broadening the lines.

The overall transmittance of each sample is an interplay of substrate and print quality of the specific ink. Samples having flexible thin glass as substrate show highest transmittance of approximately $75 \%$ for high conductive samples. Print quality was better and the effect of toning was absent even for smallest Ag nanoparticles.

In the second part of the paper, a software package was introduced that allows to simulate the electrical properties of the printed grids. For two exemplary device architectures, namely an OLED and a PV cell, artificial load layers were integrated into the device structure. It was shown that the poor design of 
the grid geometry is detrimental for device performance. The examples show how simulations can be deployed to design and optimize grid electrodes for a specific application.

\section{Acknowledgements}

We thank Dr. R. Brönnimann (Empa) for help with conductivity measurement. Funding is acknowledged from Empa and the Coating Competence Center at Empa.

\section{References}

1. Lee $H B$, Jin $W-Y$, Ovhal MM, et al (2019) Flexible transparent conducting electrodes based on metal meshes for organic optoelectronic device applications: a review. J Mater Chem C 10871110. https://doi.org/10.1039/C8TC04423F

2. Zhang F, Johansson M, Andersson MR, et al (2002) Polymer photovoltaic cells with conducting polymer anodes. Adv Mater 14:662-665. https://doi.org/10.1002/15214095(20020503)14:9<662::AID-ADMA662>3.0.CO;2-N

3. Jung $\mathrm{H}, \mathrm{Yu}$ JS, Lee HP, et al (2013) A scalable fabrication of highly transparent and conductive thin films using fluorosurfactant-assisted single-walled carbon nanotube dispersions. Carbon $\mathrm{N}$ Y 52:259-266. https://doi.org/10.1016/j.carbon.2012.09.027

4. Gaynor W, Hofmann S, Christoforo MG, et al (2013) Color in the Corners: ITO-Free White OLEDs with Angular Color Stability. Adv Mater 25:4006-4013.

https://doi.org/10.1002/adma.201300923

5. Gomez De Arco L, Zhang Y, Schlenker CW, et al (2010) Continuous, Highly Flexible, and Transparent Graphene Films by Chemical Vapor Deposition for Organic Photovoltaics. ACS Nano 4:2865-2873. https://doi.org/10.1021/nn901587x

6. Zhou G, Wu S, Zeng M, et al (2017) A Practical ITO Replacement Strategy: Sputtering-Free Processing of a Metallic Nanonetwork. Adv Mater Technol 2:1700061-1700066. https://doi.org/10.1002/admt.201700061

7. Kumar G, Li Y-D, Biring S, et al (2017) Highly efficient ITO-free organic light-emitting diodes employing a roughened ultra-thin silver electrode. Org Electron 42:52-58. https://doi.org/10.1016/J.ORGEL.2016.12.021

8. Lin Z, Wang J (2014) Low-cost Nanomaterials. Springer London, London

9. Morales-Masis M, De Wolf S, Woods-Robinson R, et al (2017) Transparent Electrodes for Efficient Optoelectronics. Adv Electron Mater 3:1600529-1600546.

https://doi.org/10.1002/aelm.201600529

10. Yu J, Kim I, Kim J, et al (2012) Nanoscale Silver front electrode grids for ITO-free all printed 
polymer solar cells with embedded and raised topographies, prepared by thermal imprint, flexographic. Nanoscale 4:6032-6040. https://doi.org/10.1039/c2nr31508d

11. Cui Z, Gao Y (2015) Hybrid Printing of High Resolution Metal Mesh as Transparent Conductor for Touch Panel and OLED. SID 2015 Dig 398-400

12. Kang MG, Joon Park H, Hyun Ahn S, Jay Guo L (2010) Transparent Cu nanowire mesh electrode on flexible substrates fabricated by transfer printing and its application in organic solar cells. Sol Energy Mater Sol Cells 94:1179-1184. https://doi.org/10.1016/j.solmat.2010.02.039

13. Eckstein R, Hernandez-Sosa G, Lemmer U, Mechau N (2014) Aerosol jet printed top grids for organic optoelectronic devices. Org Electron physics, Mater Appl 15:2135-2140. https://doi.org/10.1016/j.orgel.2014.05.031

14. Kopola P, Zimmermann B, Filipovic A, et al (2012) Aerosol jet printed grid for ITO-free inverted organic solar cells. Sol Energy Mater Sol Cells 107:252-258.

https://doi.org/10.1016/j.solmat.2012.06.042

15. Jeong J, Kim J, Kim H (2011) Solar Energy Materials \& Solar Cells Ag grid / ITO hybrid transparent electrodes prepared by inkjet printing. Sol Energy Mater Sol Cells 95:1974-1978. https://doi.org/10.1016/j.solmat.2011.02.016

16. Georgiou E, Choulis SA, Hermerschmidt F, et al (2018) Printed Copper Nanoparticle Metal Grids for Cost-Effective ITO-Free Solution Processed Solar Cells. Sol RRL 2:1700192-1700200. https://doi.org/10.1002/solr.201700192

17. Kim I, Kwak SW, Ju Y, et al (2015) Roll-offset printed transparent conducting electrode for organic solar cells. Thin Solid Films 580:21-28. https://doi.org/10.1016/j.tsf.2015.02.075

18. Choi Y-M, Kim K-Y, Lee E, et al (2014) Fabrication of a single-layer metal-mesh touchscreen sensor using reverse-offset printing. J Inf Disp 16:37-41. https://doi.org/10.1080/15980316.2014.991770

19. Mo L, Ran J, Yang L, et al (2016) Flexible transparent conductive films combining flexographic printed silver grids with CNT coating. Nanotechnology 27:065202-065211. https://doi.org/10.1088/0957-4484/27/6/065202

20. Pudas M, Hagberg J, Leppävuori S (2004) Printing parameters and ink components affecting ultra-fine-line gravure-offset printing for electronics applications. J Eur Ceram Soc 24:29432950. https://doi.org/10.1016/J.JEURCERAMSOC.2003.11.011

21. Wang L, Lo C (2017) Morphology and Conductivity Enhancement of Metal Mesh in OLEDs by Near Infrared and Intense Pulse Light. Proeeedings 12th IEEE Int Conf Nano/Micro Eng Mol Syst $34-37$

22. Jin W, Ginting RT, Ko K, Kang J (2016) Ultra-Smooth, Fully Solution- Processed Large-Area 
Transparent Conducting Electrodes for Organic Devices. Sci Rep 36475-36485. https://doi.org/10.1038/srep36475

23. Lee TM, Noh JH, Kwak SW, et al (2012) Design and fabrication of printed transparent electrode with silver mesh. Microelectron Eng 98:556-560. https://doi.org/10.1016/j.mee.2012.07.003

24. Visser CW, Pohl R, Sun C, et al (2015) Toward 3D Printing of Pure Metals by Laser-Induced Forward Transfer. Adv Mater 27:4087-4092. https://doi.org/10.1002/adma.201501058

25. Yi H, Qi L, Luo J, et al (2018) Elimination of droplet rebound off soluble substrate in metal droplet deposition. Mater Lett 216:232-235. https://doi.org/10.1016/J.MATLET.2018.01.127

26. Yi H, Qi L, Luo J, et al (2019) Direct fabrication of metal tubes with high-quality inner surfaces via droplet deposition over soluble cores. J Mater Process Technol 264:145-154. https://doi.org/10.1016/J.JMATPROTEC.2018.09.004

27. Cai G, Darmawan P, Cui M, et al (2016) Highly Stable Transparent Conductive Silver Grid/PEDOT:PSS Electrodes for Integrated Bifunctional Flexible Electrochromic Supercapacitors. Adv Energy Mater 6:1501882-1501890. https://doi.org/10.1002/aenm.201501882

28. Lim JW, Lee YT, Pandey R, et al (2014) Effect of geometric lattice design on optical/electrical properties of transparent silver grid for organic solar cells. Opt Express 22:26891-26899. https://doi.org/10.1364/OE.22.026891

29. Kipphan H (2001) Handbook of Print Media. Springer Berlin Heidelberg, Berlin

30. Yi H, Qi L, Luo J, Li N (2019) Hole-defects in soluble core assisted aluminum droplet printing: Metallurgical mechanisms and elimination methods. Appl Therm Eng 148:1183-1193. https://doi.org/10.1016/J.APPLTHERMALENG.2018.12.013

31. Yi H, Qi L, Luo J, et al (2018) Effect of the surface morphology of solidified droplet on remelting between neighboring aluminum droplets. Int J Mach Tools Manuf 130-131:1-11. https://doi.org/10.1016/J.IMACHTOOLS.2018.03.006

32. Yi H, Qi L, Luo J, et al (2016) Pinhole formation from liquid metal microdroplets impact on solid surfaces. Appl Phys Lett 108:041601-041606. https://doi.org/10.1063/1.4940404

33. Qi L, Li J, Zhu C, et al (2016) Realization of a flexible and mechanically robust Ag mesh transparent electrode and its application in a PDLC device. RSC Adv 6:13531-13536. https://doi.org/10.1039/C5RA21988D

34. Yi M, Yeom D, Lee W, et al (2010) Scalability of Roll-to-Roll Gravure-Printed Electrodes on Plastic Foils. IEEE Trans Electron Packag Manuf 33:275-283. https://doi.org/10.1166/jnn.2013.7060

35. Park H, Kang H, Lee $\mathrm{Y}$, et al (2012) Fully roll-to-roll gravure printed rectenna on plastic foils for wireless power transmission at $13.56 \mathrm{MHz}$. Nanotechnology 23:344006-344012. 
https://doi.org/10.1088/0957-4484/23/34/344006

36. Park J, Nguyen HA, Park S, et al (2015) Roll-to-roll gravure printed silver patterns to guarantee printability and functionality for mass production. Curr Appl Phys 15:367-376.

https://doi.org/10.1016/j.cap.2015.01.007

37. Zhang XQ, Liu K, Sunappan V, Shan X (2015) Diamond micro engraving of gravure roller mould for roll-to-roll printing of fine line electronics. J Mater Process Technol 225:337-346. https://doi.org/10.1016/j.jmatprotec.2015.05.032

38. Kim S, Sung HJ (2015) Effect of printing parameters on gravure patterning with conductive silver ink. J Micromechanics Microengineering 25:045004-045018.

https://doi.org/10.1088/0960-1317/25/4/045004

39. Sung D, De La Fuente Vornbrock A, Subramanian V (2010) Scaling and optimization of gravureprinted silver nanoparticle lines for printed electronics. IEEE Trans Components Packag Technol 33:105-114. https://doi.org/10.1109/TCAPT.2009.2021464

40. Huang J, Miller PF, de Mello JC, et al (2003) Influence of thermal treatment on the conductivity and morphology of PEDOT/PSS films. Synth Met 139:569-572. https://doi.org/10.1016/S03796779(03)00280-7

41. Sartoris G (2017) NM-SESES

42. Ruhstaller B, Buecheler S, Feurer T, Losio PA (2016) Evolutionary Optimization of TCO/Mesh Electrical Contacts in CIGS Solar Cells. 32nd Eur Photovolt Sol Energy Conf Exhib 1237-1240. https://doi.org/10.4229/EUPVSEC20162016-3CV.4.32

43. Lanz T, Bonmarin M, Stuckelberger M, et al (2013) Electrothermal Finite-Element Modeling for Defect Characterization in Thin-Film Silicon Solar Modules. IEEE J Sel Top Quantum Electron 19:1-8. https://doi.org/10.1109/JSTQE.2013.2250259

44. Burns S, Macleod J, Trang Do T, et al (2017) Effect of thermal annealing Super Yellow emissive layer on efficiency of OLEDs. Sci Rep 7:1-8. https://doi.org/10.1038/srep40805

45. Makha M, Letícia S, Jenatsch S, et al (2016) A transparent, solvent-free laminated top electrode for perovskite solar cells. Sci Technol Adv Mater 17:260-266

46. Grau G, Cen J, Kang H, et al (2016) Gravure-printed electronics: recent progress in tooling development, understanding of printing physics, and realization of printed devices. Flex Print Electron 1:023002-023025. https://doi.org/10.1088/2058-8585/1/2/023002

47. Yu P-C, Hong C-C, Liou T-M (2016) Bendable transparent conductive meshes based on multilayer inkjet-printed silver patterns. J Micromechanics Microengineering 26:035012-035019. https://doi.org/10.1088/0960-1317/26/3/035012

48. Song JH, Edirisinghe MJ, Evans JRG (2004) Formulation and Multilayer Jet Printing of Ceramic 
Inks. J Am Ceram Soc 82:3374-3380. https://doi.org/10.1111/j.1151-2916.1999.tb02253.x

49. Ju M, Lee Y-J, Lee J, et al (2012) Double screen printed metallization of crystalline silicon solar cells as low as $30 \mu \mathrm{m}$ metal line width for mass production. Sol Energy Mater Sol Cells 100:204208. https://doi.org/10.1016/J.SOLMAT.2012.01.018

50. Laaperi A (2008) OLED lifetime issues from a mobile-phone-industry point of view. J Soc Inf Disp 16:1125-1130. https://doi.org/10.1889/JSID16.11.1125 


\section{Supplementary Information}

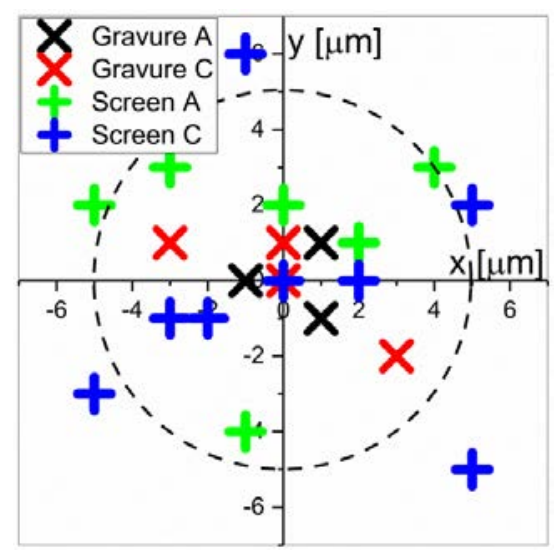

Figure S1: Overprinting accuracy of the two printing units for inter- and intra-repeated printing. The position for two markers ( $A$ and $C$ ) is shown after repeated printing. A $5 \mu \mathrm{m}$ radius circle is drawn as guide to the eye. 
Table S1: Printed grids: Data and parameter.

\begin{tabular}{|c|c|c|c|c|c|c|c|c|c|}
\hline \multirow[t]{2}{*}{ Name } & \multirow[t]{2}{*}{ Ink } & \multirow{2}{*}{$\begin{array}{l}\text { Speed } \\
{[\mathrm{mm} / \mathrm{s}]}\end{array}$} & \multirow{2}{*}{$\begin{array}{l}\text { No. of } \\
\text { Layer }\end{array}$} & \multirow{2}{*}{$\begin{array}{l}\Delta \text { Gap * } \\
{[\mathrm{mm}]}\end{array}$} & \multicolumn{2}{|c|}{ Resistivity [ $\Omega /$ sample] } & \multirow[b]{2}{*}{ C } & \multirow[b]{2}{*}{$\mathrm{D}$} & \multirow[b]{2}{*}{$E$} \\
\hline & & & & & A & B & & & \\
\hline PET1 & $20 \mathrm{~nm}$ & 300 & 1 & 0.06 & 2400 & 2900 & 1600 & 2300 & 370 \\
\hline PET2 & $20 \mathrm{~nm}$ & 300 & 1 & 0.06 & 3300 & 1600 & 635 & 809 & 574 \\
\hline PET3 & $20 \mathrm{~nm}$ & 300 & 2 & 0.06 & 141 & 271 & 3.2 & 4.9 & 4.3 \\
\hline PET4 & $20 \mathrm{~nm}$ & 300 & 3 & 0.06 & 4.158 & 7.83 & 1.266 & 2.16 & 1.9 \\
\hline PET5 & $20 \mathrm{~nm}$ & 600 & 1 & 0.06 & 3880 & 2980 & 329 & 637 & 711 \\
\hline PET6 & $20 \mathrm{~nm}$ & 800 & 1 & 0.06 & 25000 & 1900 & 1000 & 1400 & 2500 \\
\hline PET7 & $20 \mathrm{~nm}$ & 1000 & 1 & 0.06 & 4700 & 2000 & 763 & 1200 & 1520 \\
\hline PET8 & $5 \mathrm{~nm}$ & 300 & 1 & 0.14 & 378 & 386 & 16.4 & 29 & 14.4 \\
\hline PET9 & $5 \mathrm{~nm}$ & 400 & 1 & 0.14 & 722 & 528 & 27.2 & 98 & 23.7 \\
\hline PET10 & $5 \mathrm{~nm}$ & 500 & 1 & 0.14 & 757 & 460 & 15.6 & 29.1 & 9.8 \\
\hline PET11 & $5 \mathrm{~nm}$ & 800 & 1 & 0.14 & 666 & 423 & 7.9 & 26 & 17.8 \\
\hline PET12 & $5 \mathrm{~nm}$ & 1000 & 1 & 0.14 & 704 & 514 & 11.4 & 97.3 & 64.6 \\
\hline PET13 & $5 \mathrm{~nm}$ & 1000 & 1 & 0.06 & 592 & 435 & 18.1 & 233 & 284 \\
\hline PET14 & $5 \mathrm{~nm}$ & 800 & 1 & 0.06 & 824 & 514 & 16.49 & 243 & 258 \\
\hline PET15 & $5 \mathrm{~nm}$ & 600 & 1 & 0.06 & 644 & 500 & 11.5 & 207 & 246 \\
\hline PET16 & $5 \mathrm{~nm}$ & 500 & 1 & 0.06 & 666 & 741 & 5.9 & 58 & 129.4 \\
\hline PET17 & $5 \mathrm{~nm}$ & 400 & 1 & 0.06 & 603 & 540 & 24 & 100 & 132 \\
\hline CTA & $5 \mathrm{~nm}$ & 400 & 1 & 0.06 & 2200 & 1600 & 4.2 & 169 & 1600 \\
\hline Lam1 & $5 \mathrm{~nm}$ & 800 & 2 & 0.08 & 781 & 1350 & 2.8 & 1500 & 2900 \\
\hline Lam2 & $5 \mathrm{~nm}$ & 800 & 3 & 0.08 & 5.7 & 6.9 & 1.7 & 33.3 & 54.1 \\
\hline Lam3 & $5 \mathrm{~nm}$ & 1000 & 3 & 0.08 & 14.4 & 14.7 & 2.8 & 19.3 & 3000 \\
\hline PET18 & $5 \mathrm{~nm}$ & 800 & 3 & 0.06 & 2.86 & 4.78 & 0.86 & 3.06 & 7.61 \\
\hline PET19 & $5 \mathrm{~nm}$ & 800 & 2 & 0.06 & 6.46 & 24.63 & 1.26 & 51.47 & 425 \\
\hline PET20 & $5 \mathrm{~nm}$ & 1200 & 3 & 0.06 & 3.6 & 8.2 & 1.7 & 7.4 & 71.5 \\
\hline Glass1 & $5 \mathrm{~nm}$ & 800 & 3 & 0.08 & 35.9 & 16.6 & 2.8 & 660 & 5.8 \\
\hline Glass2 & $5 \mathrm{~nm}$ & 800 & 3 & 0.08 & 9.7 & 16.1 & 2.3 & 128 & 70 \\
\hline Glass3 & $5 \mathrm{~nm}$ & 800 & 3 & 0.08 & 8.2 & 10 & 4.1 & $1 \mathrm{E}+13$ & 8.6 \\
\hline
\end{tabular}

${ }^{*} \Delta$ Gap $=(\text { Gap-Substrate })^{\star}-1$ : substrate/rubble blanket sandwich is squeezed by this value. Higher value $=$ higher pressure . 


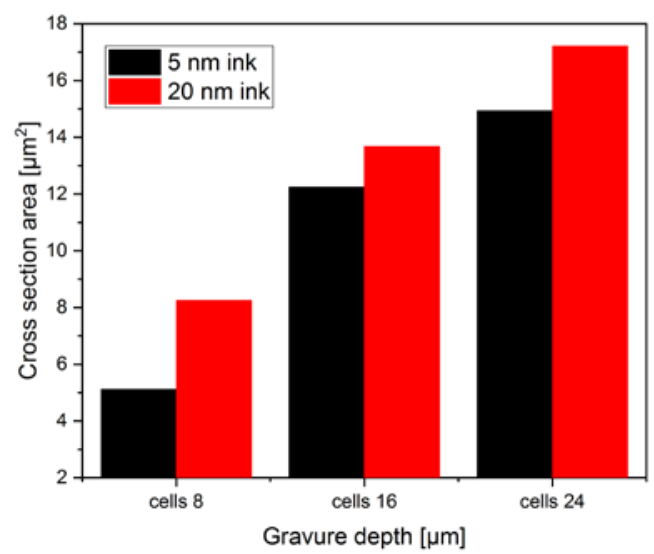

Figure S2: Cross section of the printed lines for 3 gravure cell depths and 2 used Ag inks.

Table S2: Measured line width for grids A and B and 1, 2 or 3 layers, respectively.

\begin{tabular}{|c|c|c|c|}
\hline Grid A/B & 1 Layer & 2 Layer & 3 Layer \\
\hline $5 \mathrm{~nm}$ ink & $43 \pm 2 \mu \mathrm{m}(\mathrm{PET} 14)$ & $41 \pm 2 \mu \mathrm{m}(\mathrm{PET} 19)$ & $44 \pm 2 \mu \mathrm{m}($ PET18) \\
\hline 20 nm ink & $32 \pm 2 \mu \mathrm{m}(\mathrm{PET} 2)$ & $33 \pm 2 \mu \mathrm{m}(\mathrm{PET} 3)$ & $59 \pm 2 \mu \mathrm{m} \quad(\mathrm{PET} 4)$ \\
\hline
\end{tabular}

